\title{
Correction to: Transorbital endoscopic approaches to the skull base: a systematic literature review and anatomical description
}

\author{
Alperen Vural ${ }^{1,2} \cdot$ Andrea Luigi Camillo Carobbio ${ }^{1,3,4} \cdot$ Marco Ferrari $^{2,5} \cdot$ Vittorio Rampinelli $^{2} \cdot$ Alberto Schreiber $^{2}$. \\ Davide Mattavelli $^{2}$ - Francesco Doglietto ${ }^{6}$ - Barbara Buffoli ${ }^{7}$ - Luigi Fabrizio Rodella ${ }^{7}$. Stefano Taboni ${ }^{2,5}$. \\ Michele Tomasoni ${ }^{2} \cdot$ Tommaso Gualtieri $^{2}$ - Alberto Deganello ${ }^{2} \cdot$ Lena Hirtler $^{8}$ - Piero Nicolai ${ }^{5}$
}

Published online: 14 August 2021

(C) Springer-Verlag GmbH Germany, part of Springer Nature 2021

Correction to: Neurosurg Rev https://doi.org/10.1007/s10143-020-01470-5

There was a mistake in the original article that have caused for the acknowledgment to appear incomplete. The complete acknowledgment appears below:

Acknowledgements Olympus Europa SE \& Co. KG is acknowledged for supporting the present study through provision of $4 \mathrm{~K}$ endoscopic camera and endoscopes. The Center for Anatomy and Cell Biology (Division of Anatomy) of the Medical University of Vienna is acknowledged for providing the laboratory and instrumentation for anatomical dissection.

The online version of the original article can be found at https://doi.org/ 10.1007/s10143-020-01470-5

Alperen Vural

alperenvural@yahoo.com

1 Department of Otorhinolaryngology, Erciyes University Faculty of Medicine, 38039 Kayseri, Turkey

2 Unit of Otorhinolaryngology - Head and Neck Surgery, Department of Medical and Surgical Specialties, Radiological Sciences, and Public Health, University of Brescia, Piazzale Spedali Civili 1, 25123 Brescia, Italy

3 IRCCS Ospedale Policlinico San Martino, Genoa, Italy
The Authors acknowledge MedCure's provision of anatomical specimens. The references 22, 34 and 60 were published online during the peer-review process and are therefore not included in the systematic review.

The word "toute" in the second paragraph, sentence number four of the "Introduction" section, should also be written as "route"

The original article has been corrected.

Publisher's note Springer Nature remains neutral with regard to jurisdictional claims in published maps and institutional affiliations.
4 Department of Surgical Sciences and Integrated Diagnostics (DISC), University of Genoa, Genoa, Italy

5 Section of Otorhinolaryngology - Head and Neck Surgery, Department of Neurosciences, University of Padua, Padua, Italy

6 Unit of Neurosurgery, Department of Medical and Surgical Specialties, Radiological Sciences, and Public Health, University of Brescia, Brescia, Italy

7 Section of Anatomy and Physiopathology, Department of Clinical and Experimental Sciences, University of Brescia, Brescia, Italy

8 Division of Anatomy, Center for Anatomy and Cell Biology, Medical University of Vienna, Vienna, Austria 